\title{
Exponential Decay of Correlations in a Model for Strongly Disordered 2D Nematic Elastomers
}

\author{
Y.-K. Yut and P.L. Taylor \\ Department of Physics, Case Western Reserve University, Cleveland, Ohio 44106-7079, USA \\ E.M. Terentjev \\ Cavendish Laboratory, University of Cambridge, Madingley Road, Cambridge, CB3 OHE, U.K.
}

\begin{abstract}
Lattice Monte-Carlo simulations were performed to study the equilibrium ordering in a twodimensional nematic system with quenched random disorder. When the disordering field, which competes against the aligning effect of the Frank elasticity, is sufficiently strong, the long-range correlation of the director orientation is found to decay as a simple exponential, $e^{-r / \xi}$. The correlation length $\xi$ itself also decays exponentially with increasing strength of the disordering field. This result represents a new type of behavior, distinct from the Gaussian and power-law decays predicted by some theories.
\end{abstract}

PACS 61.30.Cz, 75.50.Lk, 61.43.Bn

The effects of quenched random disorder are crucial for physical systems as diverse as random ferromagnets, vortex lattices in type-II superconductors, structural glasses, foams and liquid crystals. It is therefore of great importance to attain an understanding of the way in which the range of the ordering of such systems varies as a function of the relative strengths of the ordering and disordering fields. In this Letter we present results from a study of one simple model system. The behavior we observe is qualitatively different from any that has previously been discussed.

Strong disorder, manifesting itself in stochastic values of certain microscopic parameters, does not permit the system to achieve its ordered ground state at low temperatures. This results in a variety of glass-like structures with only short-range order, but with correlations distinctly different from those in a high-temperature liquid state. An example is the Sherrington-Kirkpatrick spin glass [1,2], which is represented theoretically by an Ising model with random exchange constants, the variance of whose fluctuations acts as a measure of the degree of disorder. When the characteristic energy of the disordering field is small, or the number density of discrete random impurities is low, the system is capable of establishing ideal ordering on a small but macroscopic length scale. On larger scales the cooperative effects of the random field may prevail over the demand for uniform thermodynamic order, and the long-range correlations decrease with distance in systems with sufficiently low space dimensionality. The possibility then exists of separating the effects of elasticity, which on a continuum level penalizes the deviations from the ground state order, from those of the continuum, coarse-grained, weak random field.

The concept of a well-established local order that is distorted on length scales larger than a characteristic coherence length $\xi_{d}$ to form textures with zero global average requires a multi-component order parameter. The simplest example is the vector order parameter of fer- romagnets, in which a dilute random array of impurities gives rise to correlated spin-glass textures 3, [4]. A system that is in many ways analogous to the ferromagnet is a nematic liquid crystal having a uniaxial tensor order parameter, where the principal axis (the director n) can form birefringent textures. Nematic elastomers are very close in their behavior to the correlated spin glasses, with network crosslinks acting as local sources of weak quenched disorder [5]. A recent analysis based on the concept of hierarchical replica symmetry breaking [6] has sugested that at long distances a quasi long-range order in vortex arrays pertains, i.e. the fluctuations diverge logarithmically and a simple power-law decay of long range correlations is predicted. This is in contrast to the general arguments of Imry and Ma [3], who predict that the long-range correlation of a continuous order parameter should decay as $\exp \left[-\left(r / \xi_{d}\right)^{4-d}\right]$ with $d$ the dimensionality of space.

All the existing continuum models are based on the notion of weak disorder, which is difficult to control parametrically. The region of applicability of such a concept could be tested experimentally, if a series of similar systems with systematically increasing random field were available. In reality, however, random magnets exist only with a limited range of possible impurities [7], and it is in general difficult to monitor the spatial variation of local magnetization in textures. Nematic elastomers offer more promise for experimental studies because the birefringent textures are readily accessible to several standard optical techniques [8]. This gives us the motivation to study such systems in depth.

In this Letter we use the direct lattice Monte-Carlo simulation of equilibrium textures in a model corresponding to nematic elastomers, which thus shows strong similarity to spin glasses. We look for the change in longrange correlations that occurs in response to a variation of random field strength. Because we need to analyze large systems, for computational efficiency we consider only the case of $d=2$. The nematic director field $\mathbf{n}(\mathbf{r})$ is 
assumed to be of constant (unit) amplitude and its direction to vary in the plane so that a single angle $\theta(x, y)$ is the only coordinate to enter the Hamiltonian. This corresponds to a locally well-established orientational order forming a large-scale texture, and is closely related to a 2D XY model $(d=2, n=2)$, the difference lying in the invariance of the nematic elastomer to inversion of the director $\mathbf{n}$ to $-\mathbf{n}$.

We consider a nematic with misoriented rod-like crosslinks, which locally impose a preferred orientation direction on the director field. This is analogous to a continuum ferromagnet with randomly distributed impurities. The Hamiltonian of such a system can be written as:

$$
\mathcal{H}=\int \frac{1}{2} K(\nabla \mathbf{n})^{2} \mathbf{d r}-\frac{1}{2} \sum_{\ell} \gamma\left(\mathbf{k}_{\ell} \cdot \mathbf{n}\right)^{2} .
$$

Here $K$ is the Frank elastic constant (in the singleconstant approximation) and $\gamma$ the strength of the randomly oriented field, whose local direction is defined by the unit vector $\mathbf{k}_{\ell}$ at the crosslink location $\ell$. In a typical elastomer the crosslinks are separated by distances much smaller than the expected correlation length $\xi_{d}$, and typically of order $1-5 \mathrm{~nm}$.

To perform the Monte Carlo study we first make the assumption that the essential element of the disorder lies in the orientational randomness of the crosslinks rather than their spatial distribution. This allows us to use a model in which the sites $\ell$ form a regular lattice, each site of which contains a random field $\mathbf{k}(\ell)$, with no correlations between neighbors. The density of crosslinks, $\rho_{0}$, is thus assumed uniform.

The minimization of the free energy (1) determines in each cell the local director orientation $\mathbf{n}\left(\mathbf{r}_{\ell}\right)$, which at zero temperature will be a function only of the dimensionless parameter $D^{*} \equiv 4 \gamma / K$. The resulting discrete dimensionless Hamiltonian, scaled by the Frank constant $K$, takes the form

$$
\mathcal{H}=\sum_{\ell}\left[-\sum_{\delta} \cos ^{2}\left(\theta_{\ell}-\theta_{\ell+\delta}\right)-\frac{1}{2} D^{*}\left(\mathbf{k}_{\ell} \cdot \mathbf{n}_{\ell}\right)^{2}\right],
$$

where $\delta$ are the nearest-neighbor lattice vectors, and the lattice spacing, $\rho_{0}^{-1 / 2}$, becomes the unit of length.

The key information we seek is the form of the correlation function $C(\mathbf{r})$ that describes the distance over which the director remains largely unchanged in orientation. In the systems we shall study, in which the director is confined to a 2-dimensional manifold, this requires computation of the average over positions $\mathbf{r}_{\mathbf{0}}$ of $C(\mathbf{r})=2\left\langle\cos ^{2}\left[\theta\left(\mathbf{r}+\mathbf{r}_{\mathbf{0}}\right)-\theta\left(\mathbf{r}_{\mathbf{0}}\right)\right]\right\rangle-1$. For a macroscopically isotropic (polydomain) system we expect this quantity to be a function only of $|\mathbf{r}|$, and so we have chosen to evaluate it as the average over all directions of $\mathbf{r}$.

Rather than perform the simulations on a very large system we studied lattices of modest size (typically $60 \times$ $60)$ and performed many repeated evaluations of $C(\mathbf{r})$ for different realizations of the random array of crosslink orientations. In this way it was possible to estimate the reliability of the results from the width of the spread in $C(\mathbf{r})$ produced. The effect of the lattice size was found by comparing the results for lattices of $60 \times 60$ and $40 \times 40$ sites. The values of $C(\mathbf{r})$ were indistinguishable for small $|\mathbf{r}|$, and differed by only a few per cent even when $|\mathbf{r}|$ was half the size of the smaller lattice.

The procedure adopted to find the minimum energy configuration was to run the Metropolis algorithm for the Monte Carlo method at a finite temperature and then to reduce the temperature to zero for the remainder of the run. This process of annealing and quenching avoided having the system be stranded in some of the smaller local energy minima. Some further tests of the robustness of the results were made by varying the initial conditions. A starting point of complete alignment, in which the directors at each lattice site were initially mutually parallel, produced results for $C(\mathbf{r})$ that tended to be larger than when the starting point was a completely random set of orientations. Each series of runs was then continued until the results from the two opposing starting points converged onto each other.

A more physically significant choice to be made concerned the boundary conditions used. The evaluation of a reliable form for $C(\mathbf{r})$ is greatly facilitated by using periodic boundary conditions, since then all lattice points are equivalent. On the other hand, the absence of any physical boundary to the system has the consequence that topological defects cannot evaporate at a surface, and can only be eliminated by a process of mutual annihilation. The weakness of the effective attraction that occurs between certain types of defects hinders this process, and makes some defects very long lived. The presence of a free boundary thus greatly speeds the process of equilibration, as defects are able simply to wander off the edge of the system. The disadvantage that comes with the use of free boundaries, however, is the fact that not all lattice sites are equivalent, and the calculation of correlation functions must be limited to pairs of sites that are in the interior of the system. In the simulations to be described below, it was indeed found that the use of free boundary conditions led to a greatly accelerated approach to equilibrium, and so this scheme was generally employed.

The decision to adopt free boundary conditions was reinforced by an examination of the consequences of applying a boundary condition intended to represent the effects of surface anchoring. In this modification, a uniform field was applied only to the lattice sites lying at the boundaries, and the simulation was run with the two opposing initial configurations, the totally random and the completely aligned. It was then found that the two sets of correlation functions never approached each other, no matter how long the run was continued, the initially aligned state giving a larger value for the correlation function at all separations $\mathbf{r}$ than the one starting from totally random director field. To avoid this effect 
we have preferred the free boundary conditions.

The correlation function $C(r)$ was calculated as the average of $2\left(\mathbf{n}_{\ell_{0}} \cdot \mathbf{n}_{\ell_{0}+\ell}\right)^{2}-1$ over all sites $\ell$ contained within a circular shell of radius $r$, centered on site $\ell_{0}$ and of thickness one lattice spacing. The calculations thus describe the effects of the competition between the random field, which tends to destroy all correlations, and the nematic interaction, which tends to introduce long-range correlations in the director orientation.

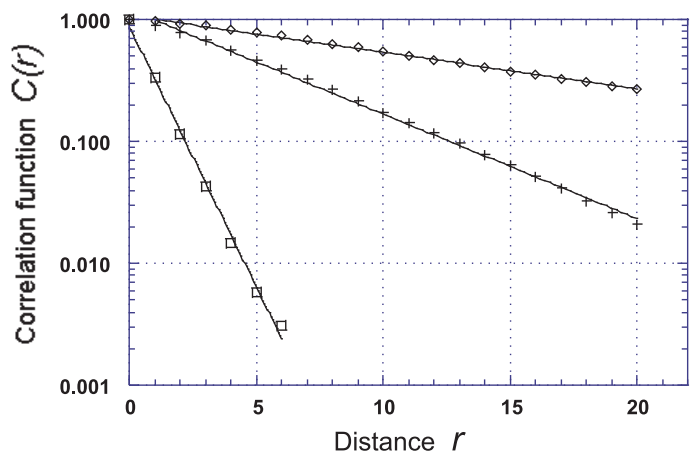

FIG.1 The orientation correlation function $C(r)$ is shown as a function of distance, measured in lattice spacings, for values of $D^{*}$, the relative strength of the random field due to crosslinks, equal to 0.5 (diamonds), 1.0 (crosses), and 3.0 (squares).

When $D^{*}$ is large, the effect of the random fields of the crosslinks dominates, and the decay of $C(r)$ is extremely rapid. As $D^{*}$ is reduced, the range of the correlation function gradually increases. In the range studied, for which $0.5<D^{*}<3.5$, the correlation function is found to approximate fairly closely a simple exponential. This is illustrated in Fig. , which shows on a semilogarithmic plot the form of $C(r)$ for $D^{*}=0.5,1.0$, and 3.0 with lines representing the exponential fit.

The necessity of checking the robustness of the calculation by choosing two opposite starting configurations was confirmed by performing an average of 50 runs of 75 000 time steps at each of the 3600 sites, starting from both ordered and random initial states. The two results were in disagreement by about $30 \%$ at large $r$. Only after the runs had been continued to 150000 time steps did the two curves approach each other sufficiently to be indistinguishable.

The linearity of the plots in Fig. tells us that the correlation function can be written in the form $C(r)=e^{-r / \xi}$ over the range of $D^{*}$ studied. This form for the correlation function permits us to identify $\xi\left(D^{*}\right)$ as the correlation length for this system, measured in units of the lattice spacing, $\rho_{0}^{-1 / 2}$. We expect it to be related to the "domain size" 3. 3. When we examine the variation of $\xi\left(D^{*}\right)$ with $D^{*}$ we find that this in turn is fitted by an exponential to within the precision of our computations, as is shown in Fig., and thus is of the form $\xi\left(D^{*}\right) \propto e^{-D^{*} / D_{0}}$. This result is somewhat surprising in view of the fact that $\xi$ must tend to infinity as $D^{*}$ vanishes, and suggests that there are two distinct regimes for the correlation behavior in this randomly disordered nematic system. It is unfortunate that the amount of computation needed to produce reliable results increases very steeply as $D^{*}$ is reduced below 0.5 , and that it was consequently not feasible to perform simulations to follow the form of $\xi\left(D^{*}\right)$ down into this small- $D^{*}$ crossover region. At the other extreme, where $D^{*}$ becomes larger than 3 , the correlation length diminishes to a fraction of a lattice spacing. (This statement is not as devoid of meaning as it appears, since it is merely a way of saying that the correlation in orientation between nearest neighbors has fallen to a number less than $e^{-1}$.)

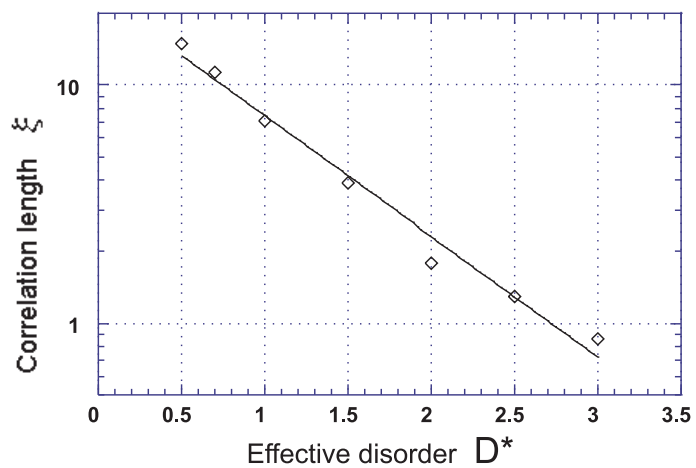

FIG.2 The effective correlation length $\xi$ is found to vary exponentially with the strength of disorder $D^{*}$.

Further calculations were made for a non-zero applied external field, which enters the Hamiltonian as $-\left(\mathbf{h} \cdot \mathbf{n}_{\ell}\right)^{2}$ and introduces an additional dimensionless parameter $\left(h^{2} / K\right)$ in Eq.(2). The results here were less unexpected, in that the correlation function $C(r)$, after an initial rapid decay, approached asymptotically a constant value that reflects the susceptibility of the system and was, accordingly, proportional to $\left(h^{2} / K\right)^{2}$ for small $h$.

To summarize, the results of our simulation describe the equilibrium ordering of nematic elastomers, whose large-scale alignment is disturbed by the quenched misorienting effect of network crosslinks. The continuum coarse-grained model used to describe this system is closely similar to that for a spin glass. We considered the case of a two-dimensional XY model, which is a good description of a thin flat sample of nematic elastomer with the director confined to this plane by planar boundary conditions. This is the typical experimental arrangement described earlier. Accordingly, the model falls into the class of $(d=2, n=2)$ continuum Hamiltonians with the quenched random field weak enough to allow formulation in terms of a continuum displacement field $\theta(x, y)$.

The classical arguments of Imry and Ma [3] predict correlations to decay as $C(r) \sim \exp \left[-\left(r / \xi_{2}\right)^{2}\right]$, while a treatment accounting for replica symmetry breaking [6] predicts $C(r) \sim(r / \xi)^{-x}$, with $x$ a positive fractional exponent. Our results appear to be quite unambiguous and to provide a totally different correlation law: $C(r) \approx \exp (-r / \xi)$ with the characteristic decay length $\xi \simeq 23 \exp \left(-1.16 D^{*}\right)$ whenever $D^{*}>0.5$. This behavior cannot persist down to vanishingly weak disorder, as it fails to predict an infinite value for $\xi$ when $D^{*} \rightarrow 0$. This 
suggests that there is a crossover to another regime at smaller values of $D^{*}=\gamma / K$ than considered here, and which might be within the range of validity of the analytical predictions [3,6]. The fact that an extrapolation of our data to $D^{*}=0$ gives a decay length roughly equal in size to the linear dimensions of the lattice used in the calculation is almost certainly only a coincidence; the large- $D^{*}$ behavior from which the extrapolation is made should be independent of the lattice size.

We are left with a number of unanswered questions. What is the physical explanation of the exponential form of the decay length? Over what range of disorder strength does it persist? Is this result unique to two dimensions or does it have a more general applicability? The resolution of these problems will require some deeper insight and a significant dedication of computational resources.

This work was made possible by NATO Collaboration Grant CRG941041, and was supported by the NSF ALCOM Science and Technology Center under Grant DMR89-20147 (PLT and YKY) and by EPSRC UK (EMT).
* Current Address: Dept. of Physics, Florida Atlantic University, Boca Raton, FL 33431

[1] D. Sherrington and S. Kirkpatrick, Phys. Rev. B 17, 4383 (1978).

[2] V.S. Dotsenko, M.V. Feigelman and L.B. Ioffe, Sov. Sci. Reviews sect.A, 15, part 1 (1990).

[3] Y. Imry and S.-K. Ma, Phys. Rev. Lett. 35, 1399 (1975).

[4] E.M. Chudnovsky, W.M. Saslow and R.A. Serota, Phys. Rev. B 33, 251 (1986).

[5] S.V. Fridrikh and E.M. Terentjev, Phys. Rev. Lett. 79, 4661 (1997).

[6] T. Giamarchi and P. Le Doussal, Phys. Rev. B 52, 1242 (1995).

[7] J. Tejada, B. Martinez, A. Labarta, E.M. Chudnovsky, Phys. Rev. B 44, 7698 (1991).

[8] J. Küpfer, H. Finkelmann, Macromol. Chem. Rapid Comm. 12, 717 (1991)

E.R. Zubarev, R.V. Talroze, T.I. Yuranova, et al, Macromol. Rapid Comm. 17, 43 (1996). S.M. Clarke, E. Nishikawa, H. Finkelmann and E.M. Terentjev, Macromol. Chem. Phys. 198, 3485 (1997);

C. Ortiz, M. Wagner, N. Bhargava, C.K. Ober, and E.J. Kramer, submitted to Macromolecules, 1997. 